\title{
Influência aguda da ordem dos exercícios resistidos em uma sessão de treinamento para peitorais e tríceps
}

\author{
Jefferson da Silva Novaes', Belmiro Freitas de Salles', Giovanni da Silva Novaes², \\ Maria Dolores Monteiro², Graça Sofia Monteiro², Miguel Videira Monteiro² \\ ${ }^{1}$ Grupo de pesquisa do Departamento de Ginástica da Escola de Educação Física \\ e Desportos da Universidade Federal do Rio de Janeiro. \\ ${ }^{2}$ Departamento de Ciência do Desporto da Universidade de Trás-os-Montes e alto Douro.
}

Novaes, J.; Salles, B.; Novaes, G.; Monteiro, M.; Monteiro, G.; Monteiro, M.; Influência aguda da ordem dos exercícios resistidos em uma sessão de treinamento para peitorais e tríceps. Motricidade 3(4): 38-45

\section{Resumo}

Este experimento investigou a Influência de diferentes ordens de exercícios resistidos (ER) em uma sessão para peitorais e tríceps sobre o número de repetições máximas (RM). Participaram 13 homens treinados $(23,8 \pm 2,5$ anos; $76,5 \pm$ $\pm 12 \mathrm{~kg} ; 178,5 \pm 6,3 \mathrm{~cm})$. Os dados foram coletados em duas fases: (a) determinação da carga para 8RM no: supino reto (SR), supino inclinado (SI) e supino declinado (SD), tríceps no pulley (TP) e tríceps na testa (TT) e (b) execução de três séries com cargas para $8 \mathrm{RM}$, três minutos de intervalo entre séries, cinco minutos entre exercícios, em duas seqüências, a saber: (SEQ1) SR, SI, SD, TP e TT; (SEQ2) TT, TP, SD, SI, e SR. Foi utilizado o teste $t$ pareado para verificar a diferença entre os números totais de RM produzidos entre seqüências. ANOVA two-way no número de RM produzidos em cada exercício entre sequências. ANOVA one-way para comparar o número de RM por série dentro de cada sequência. Para identificar estas diferenças foi utilizado o post hoc de Tukey. A significância adotada, $\mathrm{p}<0,05$. $\mathrm{O}$ número total de $\mathrm{RM}$ não apresentou diferenças significativas entre as sequências. A média do número de RM por exercício foi menor quando o mesmo foi realizado no final da sequência.

Palavras-chave: treinamento de força, exercícios resistidos, desempenho, força, ordem dos exercícios.

\section{Abstract}

Acute influence of the resistance exercise order in a training session for chest and triceps

This experiment investigated the Influence of different resistance exercise order in a training session for chest and triceps on the number of maximum repetitions (RM). Thirteen trained men participated in the experiment $(23.8 \pm 2.5$ years; $76.5 \pm 12 \mathrm{~kg} ; 178.5 \pm 6.3 \mathrm{~cm})$. Data were collected in two phases: (a) determination of a 8RM load in the: bench press (BP), incline bench press (IBP), decline press (DP), triceps pushdown (TP) and triceps extension (TE), and (b) execution of 3 sets with loads for 8RM, three minutes of interval between sets, five minutes among exercises, in two sequences, to know: (SEQ1); BP, IBP, DP, TP, and TE (SEQ2) TE, TP, DP, IBP, and BP. The paired test $t$ was used to verify the difference between the total numbers of RM produced between sequences. ANOVA two-way was used to verify the differences in the number of $\mathrm{RM}$ in each exercise among the sequences. ANOVA one-way to compare the number of RM for set inside the sequences. To identify these differences the post hoc of Tukey was used. Level significance adopted $\mathrm{p}<0.05$. The total number of RM didn't present significant differences among the sequences. The average number of RM for exercise was smaller when the same was accomplished in the end of the sequence.

Keywords: strength training, resistance exercise, performance, strength, exercise order. 


\section{Introdução}

$\mathrm{O}$ treinamento de força (TF) tornou-se uma das atividades físicas mais populares devido sua eficácia no desenvolvimento da força, hipertrofia, potência e resistência muscular. Atualmente o TF é recomendado tanto para condicionamento de atletas como para a melhora física de não atletas. $\mathrm{O}$ seu reconhecimento científico se deu a partir do posicionamento do American College of Sports Medicine de 1990 intitulado "The Recommended Quantity and Quality of Exercise for Developing and Maintaining Cardiorespiratory and Muscular Fitness in Healtby Adults", no qual, definitivamente foram incorporados os exercícios resistidos (ER) aos programas de atividades físicas objetivando a saúde.

Nos últimos anos, houve um aumento significativo de investigações científicas acerca das variáveis envolvidas no TF. Estas investigações preencheram de certa forma determinadas lacunas, entretanto muitas vezes levam à propostas questionáveis e evidências pouco conclusivas em relação à manipulação de algumas variáveis de prescrição ${ }^{2}$. Para um treinamento eficiente e seguro, é de fundamental importância um bom entendimento sobre essas variáveis. Dentre elas a intensidade de carga, o número de séries, o intervalo entre séries, a ordem dos exercícios e a velocidade de execução podem ser destacadas como algumas das mais importantes mencionadas no posicionamento oficial do $\mathrm{ACSM}^{2}$.

A ordem dos exercícios refere-se à seqüência adotada em uma sessão de treinamento ${ }^{3,45}$. De forma geral, recomenda-se que exercícios envolvendo maiores grupamentos sejam realizados no inicio de uma sessão de treinamento ${ }^{2,6,7}$. Entretanto, apenas o experimento de Sforzo \& touey ${ }^{8}$ pode fundamentar essas recomendações. Neste experimento os autores demonstraram que o volume total de repetições em uma sessão que seguia dos grandes para os pequenos grupamentos foi maior do que o volume total de uma sessão que seguia a ordem inversa. Apesar disso, pode ser observado em experimentos posteriores $^{3,4,5,9}$ que independente do tamanho do grupo muscular treinado no final de uma sessão o número de repetições sofre uma redução, não havendo diferenças significativas no volume total de repetições.

Sendo assim, algumas questões sobre a ordem dos exercícios no TF ainda permanecem inconclusivas e em alguns casos até desconhecidas. Isso ocorre no caso de uma sequência cumulativa de exercícios que envolvem os grupamentos peitorais e tríceps comumente realizados em uma mesma sessão de treinamento. O objetivo deste presente experimento foi verificar a influência de diferentes ordens de ER em uma sessão de treinamento para peitorais e tríceps, sobre o número de repetições máximas (RM) em homens treinados.

\section{Metodologia}

\section{Amostra}

Foram voluntários para o experimento, 13 indivíduos (23,8 $\pm 2,5$ anos; $76,5 \pm 12 \mathrm{~kg} ; 178,5 \pm 6,4 \mathrm{~cm})$ experientes em TF por no mínimo dois anos e frequência mínima de três vezes por semana. Antes da coleta de dados, os voluntários responderam ao questionário PAR-Q e assinaram um termo de participação consentida, conforme Resolução 196/96 do Conselho Nacional de Saúde. O Projeto de Pesquisa foi aprovado pelo comitê de ética da Universidade Federal do Rio de Janeiro. Foram excluídos do experimento indivíduos usuários de medicamentos sejam estes em prol da saúde ou em benefício do desempenho (recursos ergogênicos) e indivíduos que apresentaram qualquer tipo de limitação articular ou problemas osteomioarticulares que pudessem influenciar a realização dos exercícios propostos. 
Influência aguda da ordem dos exercícios resistidos em uma sessão de treinamento para peitorais e tríceps Jefferson da Silva Novaes, Belmiro Freitas de Salles, Giovanni da Silva Novaes, Maria Dolores Monteiro, Graça Sofia Monteiro, Miguel Videira Monteiro

\section{Procedimentos}

Os indivíduos foram submetidos a seis sessões de avaliações, quatro delas destinadas a determinação da carga para 8RM e duas para a aplicação do protocolo de treinamento. Os testes para a determinação da carga de 8RM foram divididos em duas sessões para o teste e duas sessões para o reteste. Na primeira sessão foi realizado o teste para os grandes grupos musculares, na seguinte sequência: supino reto (SR), supino inclinado (SI) e supino declinado (SD). Na segunda sessão, após 24 horas, o teste foi para os pequenos grupos, na seguinte sequência: tríceps no pulley (TP) e tríceps na testa (TT). Após 72 horas da primeira sessão foi realizada terceira sessão com o reteste para os grandes grupos. A quarta sessão foi realizada 24 horas após a terceira com o reteste para os pequenos grupos.

Finalizada a determinação da carga de 8RM através dos testes e retestes, foi respeitado um período de 48 horas para a aplicação dos protocolos de treinamento.

\section{Teste e reteste de $8 \mathrm{RM}$}

O teste de 8RM recebeu a seguinte determinação:

a) Primeiro dia: Aquecimento específico (12 repetições com cargas leves) somente para o primeiro exercício. Os exercícios seguiram a seguinte ordem: SR, SI e SD;

b) Segundo dia: Aquecimento específico (12 repetições com cargas leves) somente para o primeiro exercício. Os exercícios seguiram a seguinte ordem: TP e TT.

Visando reduzir a margem de erro no teste de 8RM, foram adotadas as seguintes estratégias: a) instruções padronizadas e familiarização antes do teste, de modo que todo o avaliado estivesse ciente de toda a rotina que envolvia a coleta de dados; b) o avaliado foi instruído sobre técnicas de execução do exercício; c) o avaliador estava atento quanto à posição adotada pelo praticante no momento da medida, pois pequenas variações do posicionamento das articulações envolvidas no movimento poderiam acionar outros músculos, levando a interpretações errôneas dos escores obtidos; d) estímulos verbais foram realizados a fim de manter alto o nível de estimulação; e) os pesos adicionais utilizados no estudo foram previamente aferidos em balança de precisão (Filizola). Os intervalos entre as tentativas em cada exercício durante o teste de 8RM foram fixados entre dois e cinco minutos. Após obtenção da carga do exercício, um intervalo não inferior a cinco minutos foi dado, antes de passar ao exercício seguinte.

Considerou-se como a carga de 8RM, o maior peso obtido em ambos os dias (teste e reteste) com diferença menor que 5\%. No caso de diferença maior, os sujeitos deveriam comparecer ao local mais uma vez para a realização de novo teste, para que o cálculo da diferença fosse refeito. Nos intervalos entre as sessões dos testes, não foi permitida a realização de exercícios que pudessem interferir nos resultados obtidos.

\section{Procedimentos}

O protocolo de treinamento consistia de duas sessões para cada voluntário, com 48 horas de intervalo entre as sessões. Os voluntários foram divididos aleatoriamente em dois grupos. $\mathrm{Na}$ primeira sessão de treinamento do grupo 1 (G1) foi escolhida a sequência dos grandes para os pequenos grupos musculares (sequência 1): SR, SI e SD, TP e o TT. E para o grupo 2 (G2), também na primeira sessão do treinamento, foram utilizados primeiro os exercícios para os pequenos grupamentos musculares, seguindo a sequência inversa (sequência 2) TT, TP, SD, SI, e SR. Após o intervalo de 48 horas, na segunda sessão de treinamento, os participantes dos dois grupos realizaram a seqüência inversa a utilizada na primeira sessão. 
Gráfico 1: Números de RM em cada exercício (média das três séries) nas duas sequências.

* Diferenças significativas $(p<0,05)$.

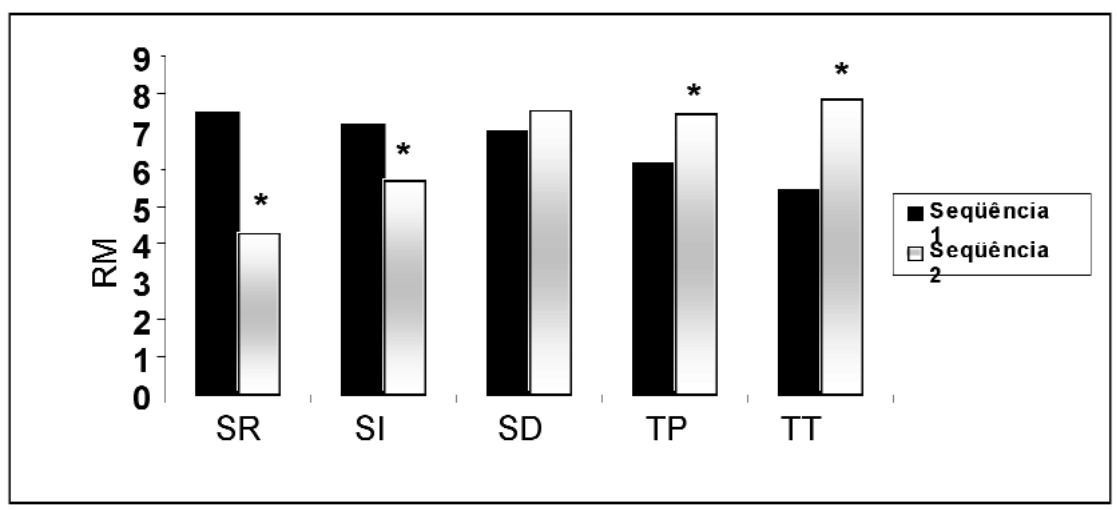

O aquecimento foi efetivado em 12 repetições com $40 \%$ da carga de $8 \mathrm{RM}$ apenas para o primeiro exercício de cada sequência. Os indivíduos deveriam realizar o exercício até a falha muscular concêntrica, sem alterar o padrão do movimento. Foram adotados três minutos de recuperação entre as séries e cinco minutos entre os exercícios. O número de RM foi registrado ao final de cada uma das três séries para os exercícios propostos em ambas as sequências.

\section{Estatística}

Para o tratamento estatístico Foi utilizado o teste $t$ pareado para verificar a diferença entre os números totais de $\mathrm{RM}$ produzidos entre sequências. ANOVA two-way no número de RM produzidos em cada exercício (média das três séries) entre sequências. ANOVA one-way foi utilizado para comparar o número de RM por série dentro de cada sequência. Para identificar estas diferenças foi utilizado o post hoc de Tukey. Também utilizamos o teste-t pareado para verificar a diferença entre os testes em 1RM, bem como o Coeficiente de Correlação Intra-classe. O nível de significância adotado foi $\mathrm{p}<0,05$, para tal foi utilizado o software Statistica versão 7.0.

\section{Resultados}

O número total de $\mathrm{RM}$ não apresentou diferenças significativas entre as sequências. Os números de RM produzidos em cada exercício (média das três séries) nas duas sequências podem ser vistos no gráfico 1 . Nele pode-se observar que o SR e o SI apresentaram médias significativamente maiores na sequência 1 , enquanto o TP e o TT apresentaram médias significativamente maiores na sequência 2 . O SD não apresentou diferença significativa entre as sequências $(p<0,05)$.

As médias dos números de RM produzidos nas três séries de cada exercício para as duas seqüências podem ser vistas na tabela 1. Nela, podem-se observar diferenças significativas entre os números de RM: no exercício SR, da terceira em relação à primeira série na sequência 1 e da terceira em relação a primeira e segunda série na sequência 2; no exercício SI, da segunda em relação a primeira série e da terceira em relação a primeira e segunda série na sequência 1 e da terceira em relação a primeira série na sequência 2; no exercício SD, da terceira em relação a primeira série apenas na sequência 1 ; e no exercício TT, da terceira em relação a primeira série em ambas as sequências. No exercício TP não foram observadas diferenças significativas entre as séries $(\mathrm{p}<0,05)$. 
Influência aguda da ordem dos exercícios resistidos em uma sessão de treinamento para peitorais e tríceps Jefferson da Silva Novaes, Belmiro Freitas de Salles, Giovanni da Silva Novaes, Maria Dolores Monteiro, Graça Sofia Monteiro, Miguel Videira Monteiro

Tabela 1: Números de RM produzidos nas três séries de cada exercício para as duas sequências (média e desvio padrão).

Exercícios

Sequência 1

Sequência 2

\begin{tabular}{|c|c|c|c|c|c|c|}
\hline & 1. Série & 2. a Série & 3. a Série & 1. Série & 2. Série & 3. a Série \\
\hline $\mathrm{SR}$ & $8,08 \pm 0,27$ & $7,62 \pm 0,65$ & $6,85 \pm 1,4^{*}$ & $5,23 \pm 0,92$ & $5,23 \pm 0,92$ & $3,15 \pm 1,34^{\star \star+}$ \\
\hline إ & $7,92 \pm 0,94$ & $7,23 \pm 0,59^{*}$ & $6,46 \pm 0,77^{\star+}$ & $6,38 \pm 1,32$ & $6,38 \pm 1,32$ & $4,85 \pm 1,40^{*}$ \\
\hline & $7,69 \pm 0,48$ & $7 \pm 0,70$ & $6,31 \pm 1,18^{*}$ & $7,92 \pm 1,03$ & $7,92 \pm 1,03$ & $7,15 \pm 1,40$ \\
\hline & $6,62 \pm 1,75$ & $6,23 \pm 1,48$ & $5,54 \pm 1,98$ & $7,85 \pm 1,21$ & $7,85 \pm 1,21$ & $7,08 \pm 1,18$ \\
\hline$[7$ & $6,08 \pm 1,84$ & $5,62 \pm 1,04$ & $4,54 \pm 1,19^{*}$ & $8,31 \pm 0,75$ & $8,31 \pm 0,75$ & $7,31 \pm 1,03^{\star}$ \\
\hline
\end{tabular}

* Diferenças significativas em relação a 1. a série do mesmo exercício e sequência $(p<0,05)$

+ Diferenças significativas em relação a 2. ${ }^{a}$ série do mesmo exercício e sequência $(p<0,05)$.

\section{Discussão}

Uma importante informação para verificar se a carga em um exercício é realmente máxima é conhecer se essa carga é reprodutível. Por isso, antes de testar o efeito de diferentes ordenações de exercícios no desempenho da força, verificamos a reprodutibilidade para as cargas obtidas em 8RM nas situações de teste e re-teste. Os coeficientes de correlação intra-classe mostraram-se elevados em todos os exercícios testados. Um dos aspectos que pode ter influenciado esses resultados foi o fato dos integrantes da amostra serem treinados em realizar exercícios com 8RM. Além disso, os exercícios selecionados faziam parte da rotina habitual de treinamento. A reprodutibilidade das cargas nos exercícios investigados assegurou a qualidade dos dados para a condução das sessões de treinamento nas diferentes sequências adotadas.

Os nossos principais resultados demonstraram que o número total de RM não apresentou diferenças significativas entre a sequência que seguia dos peitorais para os tríceps e a sequência que seguia dos tríceps para os peitorais. Pode-se verificar que ambos os grupos musculares quando treinados no final da sessão de treinamento tiveram uma tendência à diminuição em sua produção de força em relação aos grupos treinados no início, independente do seu tamanho. O SR e o SI tiveram suas médias de RM por série diminuídas em $43 \%$ e $22 \%$ respectivamente quando eram colocados no final da sessão, e o TT e o TP $31 \%$ e $18 \%$ respectivamente quando realizados como últimos exercícios. O SD por se posicionar no meio da sessão nas duas sequências utilizadas não apresentou diferenças.

Simão et al. ${ }^{4}$ obtiveram resultados similares em relação ao exercício desenvolvimento (DES) para ombros em duas ordenações diferentes quando o mesmo se apresentava no meio da sessão. Neste experimento ${ }^{4}$, os exercícios utilizados foram: SR, puxada pela frente (PF), DES, bíceps rosca (BR) e TP. Foram realizadas duas seqüências de treinamento, a sequência $A: S R$, PF, DES, BR e TP; e a seqüência B: TP, BR, DES, PF e SR. Durante ambas as sequências, foram realizadas três séries de cada exercício até a falha concêntrica e foram dados dois minutos de intervalo entre as séries. Como dito, dentre os exercícios apenas o DES não apresentou diferenças significativas em relação ao número de RM entre as seqüências. Já os outros exercícios, apresentaram diferenças significativas. Tanto os 
grandes quanto os pequenos grupamentos, quando foram posicionados ao final das sequências, resultaram em um número menor de $\mathrm{RM}$, principalmente nas últimas séries de cada exercício. Apesar disso, o número total de RM não variou.

Em experimento realizado por Monteiro et al. ${ }^{9}$ foram utilizados apenas três exercícios envolvendo os peitorais, os ombros e os tríceps. $\mathrm{Na}$ sequência A realizaram-se: SR, DES e TP enquanto na seqüência B: TP, DES e SR. Os resultados obtidos corroboraram quase que integralmente com os resultados do nosso experimento e também com os resultados apresentados por Simão et al. ${ }^{4}$. No entanto, no experimento de Monteiro et al. ${ }^{9}$ o DES mesmo posicionado no meio da sequência para as duas ordens utilizadas apresentou diferença estatística para o número de RM. Os autores relacionaram essa diferença ao fato do exercício SR exigir recrutamento de unidades motoras da cintura escapular, utilizadas também durante o DES, o que pode levar a uma maior fadiga ao executar-se o DES após o SR. No nosso experimento a fadiga acumulada durante a realização dos exercícios para peitorais SR e SI pode ter comprometido o desempenho durante a realização do exercício SD sequencialmente, já que estes exercícios envolvem o mesmo grupamento. Apesar disso, o número de RM no SD, como dito anteriormente, não apresentou diferenças significativas entre sequências. Isso pode ter acontecido porque na sequência dos pequenos para os grandes grupamentos $O$ exercício SD, que também envolve os tríceps, pode ter sido comprometido pela fadiga acumulada durante a realização dos exercícios TP e TT assim como ocorrido na outra sequência em relação aos exercícios SR e SI.

A diminuição no número total de RM quando um exercício é precedido por exercícios que envolvem a mesma parte de corpo parece ser em parte um resultado da fadiga crescente com a progressão de sessão de treinamento ${ }^{5}$. Simão $e t$ al. ${ }^{5}$ em duas sequências distintas de exercícios (sequência A: SR, DES, TP, leg press (LEG), cadeira extensora (EXT), e cadeira flexora (FLEX), e sequência B: FLEX, EXT, LEG, TR, DES, e SR demonstram que exercícios para membros inferiores e superiores envolvendo pequenos ou grandes grupamentos quando foram precedidos por exercícios para a mesma parte do corpo, o número total de RM realizado nas três séries desse exercício diminuiu. No experimento de Simão et al. , $^{5}$ os exercícios e as sequências adotadas são semelhantes às utilizadas por Sforzo e Touey ${ }^{8}$. Porém, podem ser destacadas três diferenças metodológicas: a intensidade de carga, o intervalo entre as séries, e a amostra. No experimento de Simão $e t$ al. ${ }^{5}$ foram utilizadas cargas para $80 \%$ de $1 \mathrm{RM}$ até a falha concêntrica, com dois minutos de intervalo, e a amostra era composta por mulheres. Enquanto no experimento de Sforzo e Touey ${ }^{8}$ os participantes eram homens que utilizaram cargas de 8RM, intervalos de dois minutos entre séries, três entre exercícios, e cinco entre membros superiores e inferiores. No experimento de Sforzo e Touey ${ }^{8}$, quando os grupamentos maiores se apresentaram no início da sessão (antes dos menores) foi observado um volume total de RM maior em relação à ordem inversa, enquanto Simão et al..$^{5}$ não observou diferenças no volume total de RM entre as sequências. Apesar disso, os experimentos corroboram que exercícios para membros inferiores e superiores envolvendo pequenos ou grandes grupamentos quando precedidos por um exercício para mesma parte do corpo sofrem reduções no desempenho.

De forma geral, a ordem dos exercícios em uma sessão de TF é projetada seguindo dos grupamentos maiores para os menores. Teoricamente, o desempenho nas sessões em que a ordenação dos exercícios é feita dos maiores grupamentos para os menores grupamentos, permitiria um estímulo máximo em todas as musculaturas envolvidas ${ }^{6}$. Esta ordenação é reco- 
mendada pelo posicionamento oficial do $\mathrm{ACSM}^{2}$ que tem como base um único experimento realizado por Sforzo \& Touey $^{8}$ no qual foi verificada uma menor produção de força quando os pequenos grupamentos eram treinados antes dos grandes grupamentos. Apesar disso, Alguns experimentos mais recentes ${ }^{3,4,5,9}$ demonstraram que independente do tamanho do grupo muscular envolvido, os exercícios realizados ao final de uma seqüência tem seu desempenho comprometido de forma similar sem comprometimento do número total de RM, como também foi verificado no presente experimento.

Em nosso experimento, o número de RM em cada exercício sofreu reduções mesmo que não significativas com a progressão das séries em ambas as seqüências utilizando-se intervalos de três minutos. Estes achados corroboram em parte com Sforzo \& Touey $^{8}$, Monteiro et al. ${ }^{9}$ e Simão et al. ${ }^{3,45}$ que também demonstraram uma diminuição significativa da primeira para as demais séries nos exercícios e nas ordens utilizadas. Os resultados aqui apresentados indicam que independente da seqüência adotada, a média do número de RM entre as séries sofre uma perda significativa quando o regime de contração muscular é máximo e o intervalo de recuperação estipulado é de três minutos.

Experimentos que comparam diferentes tempos de intervalos entre as séries demonstraram que intervalos iguais e menores do que três minutos resultam em quedas significativas do número de RM durante a progressão das séries $^{10,11,12,13}$. No nosso experimento a utilização de três minutos de recuperação entre as séries não apresentou diferenças sobre o declínio do número de RM quando comparado a outros experimentos de ordem de exercícios que utilizaram dois minutos de recuperaçã ${ }^{4,5,8}$. Sendo assim, a influência de diferentes tempos de recuperação sobre a manutenção do número de RM em diferentes ordens de exercícios emerge como uma lacuna de conhecimento que deve ser verificada em experimentos futuros.
Exercícios para peitorais e tríceps são comumente realizados em uma mesma sessão de treinamento. Por este motivo, o presente experimento utilizou uma sequência cumulativa de três exercícios para peitorais e dois para tríceps. Isso poderia refletir em resultados diferentes dos obtidos em experimentos anteriores ${ }^{3,4,5,8,9} \mathrm{em}$ que os exercícios utilizados não seguiam uma sequência cumulativa para os grupamentos. No entanto, os achados do presente experimento ratificam que independente de se treinarem grandes ou pequenos grupos prioritariamente, o grupamento treinado ao final de uma sessão terá uma redução na produção de força, que pode ser medida através do número de RM.

Os nossos principais resultados demonstraram que o número total de RM não apresentou diferenças significativas entre a seqüência que seguia dos peitorais para os tríceps e a sequência que seguia dos tríceps para os peitorais. Pode-se verificar que o grupo muscular treinado no final das sessões de treinamento teve uma tendência à diminuição em sua produção de força, verificada através da diminuição do número de RM, em relação ao grupo treinado no início do programa, independente do seu tamanho.

Uma sessão de TF deve seguir uma sequência de execução que atenda aos objetivos e necessidades individuais de cada praticante. Logo, independente de ser um grande grupamento muscular, como o peitoral, ou pequeno, como o tríceps, se queremos priorizá-lo, devemos treiná-lo no inicio da sessão de TF. Isto diminuirá a influência negativa causada pelo aumento da fadiga, o que pode comprometer em longo prazo os maiores ganhos em força e até mesmo na hipertrofia deste grupamento.

\section{Correspondência}

Jefferson da Silva Novaes

Estrada do Rio Morto 196, bloco 05 casa 101.

Vargem Grande, Rio de Janeiro, Brasil

CEP: 22783-210

Email: jsnovaes@terra.com.br 


\section{Referências}

1. American College of Sports Medicine. Position stand on the recommended quantity and quality of exercise for developing and maintaining cardio respiratory and muscular fitness in healthy adults. Med. Sci. Sports Exerc. 22: : 265-274. 1990.

2. American College of Sports Medicine. Position Stand on progression models in resistance training for health adults. Med. Sci. Spost. Exerc. 34: 364-380. 2002.

3. Simão, R.; M. D. Polito; L. E. Viveiros e P.T.V. Farinati. Influência da manipulação na ordem dos exercícios de força em mulheres treinadas sobre o número de repetições e percepção de esforço. Rev. Bras. Ativ. Física Saúde. 7: 53-61. 2002.

4. Simão R.; P. T. Farinati; M. D. Polito; A. S. Maior e S. J. Fleck. Influence of exercise order on the number of repetitions performed and perceived exertion during resistive exercise. $J$ strength Cond. Res. 19: 152-156. 2005.

5. Simão, R.; P. T. V. Farinati; M. D. Polito; L. E. Viveiros e S. J. Fleck. Influence of exercise order on the number of repetitions performed and perceived exertion during resistance exercise in women. J. strength Cond. Res. 21: 23-28. 2007.

6. Stone, M. H.; D. Wilson; Resistive training and selected effects. Med Clin. North Am. 69: 109-122. 1985.
7. Fleck, S. J.; Kraemer, W. J. Designing Resistance Training Programs. Human Kinetics. 2004. p. 376.

8. Sforzo, G. A. e P. R. Touey. Manipulating exercise order affects muscular performance during a resistance exercise training session. $J$. Strength Cond. Res. 10: 20-24. 1996.

9. Monteiro, W.; R. Simão e P. T. V. Farinati. Manipulação na ordem dos exercícios e sua influência sobre o número de repetições e percepção subjetiva de esforço em mulheres treinadas. Rev. Bras. Med Esp. 11: 146-150. 2005.

10. Richmond, S. R.; Godard, M. P. The effects of varied rest periods between sets of failure using bench press in recreationally trained men. J. Strength Cond. Res. 18: 846-849. 2004.

11. Willardson, J. M.; Burkett, L. N. A comparison of 3 different rest intervals on the exercise volume completed during a workout. $J$. Strength Cond. Res. 19: 23-26. 2005.

12. Willardson, J. M.; Burkett, L. N. The effect of rest interval length on bench press performance with heavy vs. light load. J. Strength Cond. Res. 20: 396-399. 2006 a.

13. Willardson, J. M.; Burkett, L. N. The effect of rest interval length on the sustainability of squat and bench press repetitions. J. Strength Cond. Res. 20: 400-403. 2006b. 